\title{
Spectrophotometric analysis of the $5200 \AA$ region for peculiar and normal stars
}

\author{
Ch. Stigler ${ }^{1}$, H. M. Maitzen ${ }^{1}$, E. Paunzen ${ }^{2}$, and M. Netopil ${ }^{1,2}$ \\ ${ }^{1}$ Institut für Astrophysik der Universität Wien, Türkenschanzstr. 17, 1180 Wien, Austria \\ e-mail: hans . michael .maitzen@univie.ac .at \\ 2 Department of Theoretical Physics and Astrophysics, Masaryk University, Kotlářská 2, 61137 Brno, Czech Republic \\ e-mail: epaunzen@physics.muni.cz
}

Received 17 July 2013 / Accepted 12 January 2014

\begin{abstract}
Context. Many chemically peculiar (CP) stars, especially the magnetic CP2 stars, show a flux depression at $5200 \AA$. The $\Delta a$ photometric system takes advantage of this characteristic to detect these objects in an efficient way. In addition, it is capable of finding metal-weak, emission-type, and shell-type objects of the upper main sequence.

Aims. To compare available observations and to detect new peculiar objects, we used a spectrophotometric catalogue consisting of 1159 stars. From this catalogue, we selected 1067 objects to synthesize three different $a$ indices to find the most efficient one for further observations. In addition, we extended the analysis to stars cooler than F5.

Methods. We employed classical $\Delta a$ photometry described by Maitzen, using simulated filter curves, the spectrophotometric $\Delta a$ index by Adelman, and a modified index.

Results. Even though the accuracy of the spectrophotometry used for this investigation is significantly lower than the photometric $\Delta a$ measurements, we are able to confirm peculiarity for most of the known CP2 stars above a certain limit of $\Delta a$. We investigated 631 stars hotter than spectral type F5 to find additional that are not yet identified peculiar objects. We find that for very low mass stars (M0), the $a$ index is independent of the colour (effective temperature).

Conclusions. The $\Delta a$ photometric system is very closely correlated with the effective temperature over a wide range of the main sequence. It is able to detect any kind of peculiarity connected to the $5200 \AA ̊$ region. Especially for low-mass stars, this opens up a new possibility of detecting peculiar objects in an efficient way.
\end{abstract}

Key words. stars: chemically peculiar - stars: early-type - techniques: photometric

\section{Introduction}

Chemically peculiar (CP) stars of the upper main sequence differ in their abundances of heavy and rare-earth elements in their photosphere from normal-type objects of the same effective temperature and luminosity range. Preston (1974) divided this group into four subgroups according to their astrophysical characteristics. Since then several investigations about their local environment and stellar parameters were performed. Babcock (1947) discovered a global dipolar magnetic field in the star 78 Virginis, which was soon followed by the detection of several other similar stars. Therefore the correlation of stellar magnetic field strengths with astrophysical processes such as diffusion and meridional circulation can be very well studied in this stellar group (Glagolevskij 2013). Another important often studied aspect is the cause of the peculiar (surface) abundances. The observed phenomenon can be explained by either classical diffusion of chemical elements depending on the balance between gravitational pull and uplift by the radiation field through absorption in spectral lines (Stift \& Alecian 2012) or selective accretion from the interstellar medium via the stellar magnetic field (Havnes \& Conti 1971). While diffusion seems to be appropriate for both magnetic and non-magnetic stars to explain spectral peculiarity, it is not yet clear to which extent the interaction with the interstellar medium via accretion and transport of angular momentum may modify the effects of diffusion and break the stellar rotational velocities during the stars' main sequence life time.

Using spectrophotometry, Kodaira (1969) was the first to notice broad-band flux depressions at 4100, 5200 and $6300 \AA$ during his investigation of the magnetic CP (CP2) star HD 221568. Photometrically, the main depressions (4100 and $5200 \AA$ ) of another CP2 star were later also found by Maitzen \& Moffat (1972) when they investigated the spectrum-variable HD 125248. This resulted in the development of the $\Delta a$ photometric system (Maitzen 1976), which makes use of the most efficient depression at $5200 \AA$. The flux depression itself is a combined contribution of $\mathrm{Si}, \mathrm{Cr}$ and $\mathrm{Fe}$ for strongly overabundant surface metallicities (Khan \& Shulyak 2007). The upper main sequence is unique for CP stars. No detailed observational analysis of this region for cooler objects has been performed so far.

In this paper, we present a study that was conducted to detect $\mathrm{CP}$ stars on the basis of spectrophotometric data for which we employed three different methods that use the flux depression at $5200 \AA$. In addition to the original method, the $a^{\prime}$ system developed by Adelman (1979) and a newly modified method were investigated. Furthermore, we investigated the capability of these systems of finding new, previously undetected, peculiar stars including metal-weak and emission-type objects of the 
upper main sequence. For the first time, the low-mass regime was searched for the behaviour of the $a$ indices in correlation with the effective temperature and luminosity.

\section{Data and reduction}

Our source of spectrophotometric data is the stellar catalogue by Kharitonov et al. (1988). It contains data for 1159 bright stars of different spectral classes in the wavelength range from 3200 to $7600 \AA$, with a spectral resolution of $50 \AA$. Each star was observed at least three times, on different nights. The root mean square (rms) relative error of the measurements is between $2 \%$ and $4 \%$ per $50 \AA$ bandpass. Stars with only two-digit measurements in the wavelength region around $5200 \AA$ were not considered in the analysis because of the statistical inaccuracy of these measurements. We also excluded early-type supergiants because of their strong photometric and spectroscopic variability. The remaining data of 1067 stars were used to synthesize different peculiarity indices. The methods used to detect CP stars are designed to have magnitudes as input. Therefore the intensity and flux values given in the catalogue were converted into magnitudes.

We are aware that there are more extensive corresponding catalogues with higher resolutions available in the literature, for example the one by Burnashev (1985). We note that this author also used at that time unpublished data by Kharitonov et al. (1988), but interpolated the data to a resolution of $25 \AA$. However, the catalogue we used is the basis of a list of spectrophotometric standards published by Glushneva et al. (1992). Therefore the quality of the data is beyond any doubt. In addition, the spectral resolution is similar to that used for the Gaia mission (Jordi et al. 2010).

Spectrophotometric observations of classical CP stars were published before, for example by Maitzen \& Muthsam (1980) and Adelman et al. (1989). However, all these analyses concentrated either on individual stars or on the comparison with results from synthetic spectra. To our knowledge, no systematic analysis of spectrophotometric observations with respect to synthesizing $\Delta a$ was conducted before.

The goal of this paper is not only to detect CP stars, but also to compare our findings with already existing $\Delta a$ photometry and spectrophotometry. For this purpose, we employed three different systems.

The $\Delta$ a photometric system by Maitzen (1976): this system compares the flux in the centre of the depression (filter $g_{2}, \lambda_{\mathrm{c}}=$ $5220 \AA$ ) with the adjacent spectral regions (filters $g_{1}, 5000 \AA$ and $y, 5500 \AA$ ) using a band-width of $130 \AA$, which represents the continuum of the star. With these measurements the index $a$ can be calculated as

$a=g_{2}-\frac{g_{1}+y}{2}$.

In principle, the positioning of the continuum filters $g_{1}$ and $y$ relative to $g_{2}$ minimizes the influence of temperature on the peculiarity index. However, due to slight wavelength mismatches of the filters, a second-order dependence on temperature is apparent in addition to a general increase of opacity around $5200 \AA$ with decreasing temperature. Therefore, one has to normalize $a$ with the index $a_{0}$ of a non-peculiar star of the same temperature (normality line), to compare different peculiar stars with each other. The photometric peculiarity index is therefore

$\Delta a=a-a_{0}$.
To do so, the corresponding filter curves had to be simulated. For simplicity, the three filters were represented by Gaussian curves with a FWHM of $130 \AA$ and a transmission maximum of $100 \%$ each. The fact that these simulated filters, in contrast to the original definition of $g_{1}, g_{2}$ and $y$, all have the same transmission curves, probably does not significantly affect $\Delta a$, because its value is always the difference between a peculiar star and the corresponding normal star with the same colour index. To convolve the Gaussian curves with the measurements of the spectrophotometric catalogue, transmission values of $50 \AA$ (bins) of the simulated filters, corresponding to the measurements with $50 \AA$ spectral resolution, had to be determined. This was achieved by segmenting the Gaussian curves into $5 \AA$ bins, which were then numerically integrated. The percentage of transmission, relative to the maximum, could now be calculated by applying the mean value theorem. For the transmission values of the $50 \AA$ bins, to be multiplied with the measurements, simply ten of the $5 \AA$ bin transmission values were summed. This approach enabled us to adapt the central wavelengths of the filters in $5 \AA$ steps to figure out the optimal filter positions. The central wavelengths of the filters were optimised with respect to a low variance of $a$ values for normal stars and secondly the highest possible $a$ values of CP2 stars. The best compromise of filter positions found for the spectrophotometric data is $5020 \AA$ for $g_{1}, 5215 \AA$ for $g_{2}$, and $5470 \AA$ for $y$. These filter positions are very close to those of the revised $\Delta a$ system by Maitzen (1980). We calculated the values of the simulated filters by multiplying the transmission values of the $50 \AA$ bins with the corresponding measurements of the catalogue and adding the results.

The $\Delta a^{\prime}$ index by Adelman (1979): it basically works in the same way as the index by Maitzen, but was especially designed for his spectrophotometric data. It is defined as

$a^{\prime}=m_{5264}-\left[m_{4785}+0.453\left(m_{5840}-m_{4785}\right)\right]$.

The measurements at 4785 and $5840 \AA$, which were both made at locations significantly bluer than $g_{1}$ and redder than $y$, represent a wavelength base for the continuum that is larger by a factor of two than in $\Delta a$ photometry. The depression itself is represented by the $5264 \AA$ value. Like the $a$ index by Maitzen, the $a^{\prime}$ index is slightly dependent on the colour of the star and therefore has to be normalized by the index of non-peculiar stars with the same colour.

The $\Delta a^{\prime}$ mod index: very early in the process of evaluating the spectrophotometric data the central wavelengths of the spectrophotometric data did not fit those of the $\Delta a^{\prime}$ index very well. To calculate the $a^{\prime}$ index, the measurements closest to the defined wavelengths were used. This resulted in inaccurate results, because the central measurement was shifted from the deepest part of the depression to the red side of the spectra and in some stars the $\mathrm{H} \beta$ line influenced the continuum value on the blue side. To address this problem two values next to each other were linearly interpolated to obtain central wavelengths closer to the predefined wavelengths of the $\Delta a^{\prime}$ index, as well as to improve the error by a factor of $\sqrt{2}$. This new index is referred to as $\Delta a^{\prime} \bmod$ and is defined as

$a^{\prime} \bmod =m_{5250}-\left[m_{4750}+0.453\left(m_{5850}-m_{4750}\right)\right]$.

The calculation of the indices $a^{\prime}$ and the $a^{\prime} \bmod$ was straightforward. They were obtained by simply applying the values of the corresponding measurements to the formula.

As next step, the indices had to be corrected with the $a$, $a^{\prime}$, and $a^{\prime}$ mod obtained from non-peculiar stars of the same 
Ch. Stigler et al.: Spectrophotometric analysis of the $5200 \AA$ A region for peculiar and normal stars

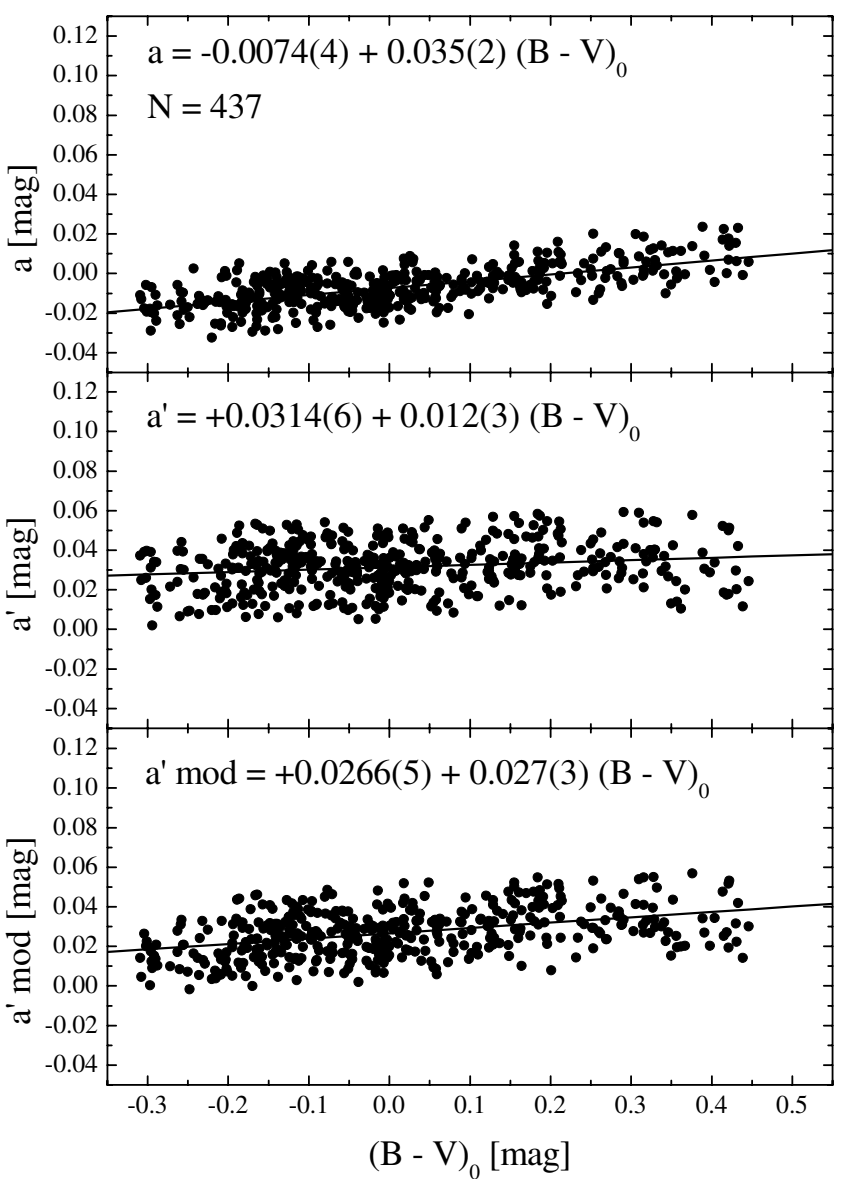

Fig. 1. Regression of 437 normal stars with reference to the different $a$ indices.

temperature. For this purpose, all stars hotter than F5 were selected to calculate $a_{0}, a_{0}^{\prime}$ and $a_{0}^{\prime} \bmod$.

According to Maitzen (1976), the indices are expected to be very well correlated with the colour index $(B-V)_{0}$. To deredden the programme stars, we made use of photometric data in the Johnson, Geneva, and Strömgren systems, compiled from the General Catalogue of Photometric Data (GCPD; Mermilliod et al. 1997). For the first two systems, the well-known calibrations based on the X/Y parameters (Cramer 1999) and Q-index (Gutierrez-Moreno 1975), respectively, which are applicable for O/B type stars were applied. Objects with available Strömgren data were treated with the routines by Napiwotzki et al. (1993), which allow dereddening of cooler-type stars. For about $60 \%$ of the targets we were able to determine reddening values, transformed to $E(B-V)$ with the ratios summarised by Netopil et al. (2008). If several estimates for a particular object were available, a mean value was calculated. Since all objects are rather bright and therefore close-by, a strong reddening especially for cool-type stars is hardly expected. We therefore adopted nonreddening for these when no Strömgren photometry was available, which is justified by the obtained overall mean $E(B-V)=$ $0.02(6) \mathrm{mag}$.

To find the normality lines, we performed an iterative linear regression of $a, a^{\prime}$ and $a^{\prime} \bmod$ versus $(B-V)_{0}$. In each iterative step, we discarded the outliers that lay more than $5 \sigma$ from the normality line. After three iterations, the errors did not significantly decrease any more. In total, we defined the normality lines from 437 stars and obtained a $3 \sigma$ of 23,36 , and $32 \mathrm{mmag}$, respectively. Figure 1 shows the regression of the peculiarity indices versus $(B-V)_{0}$.

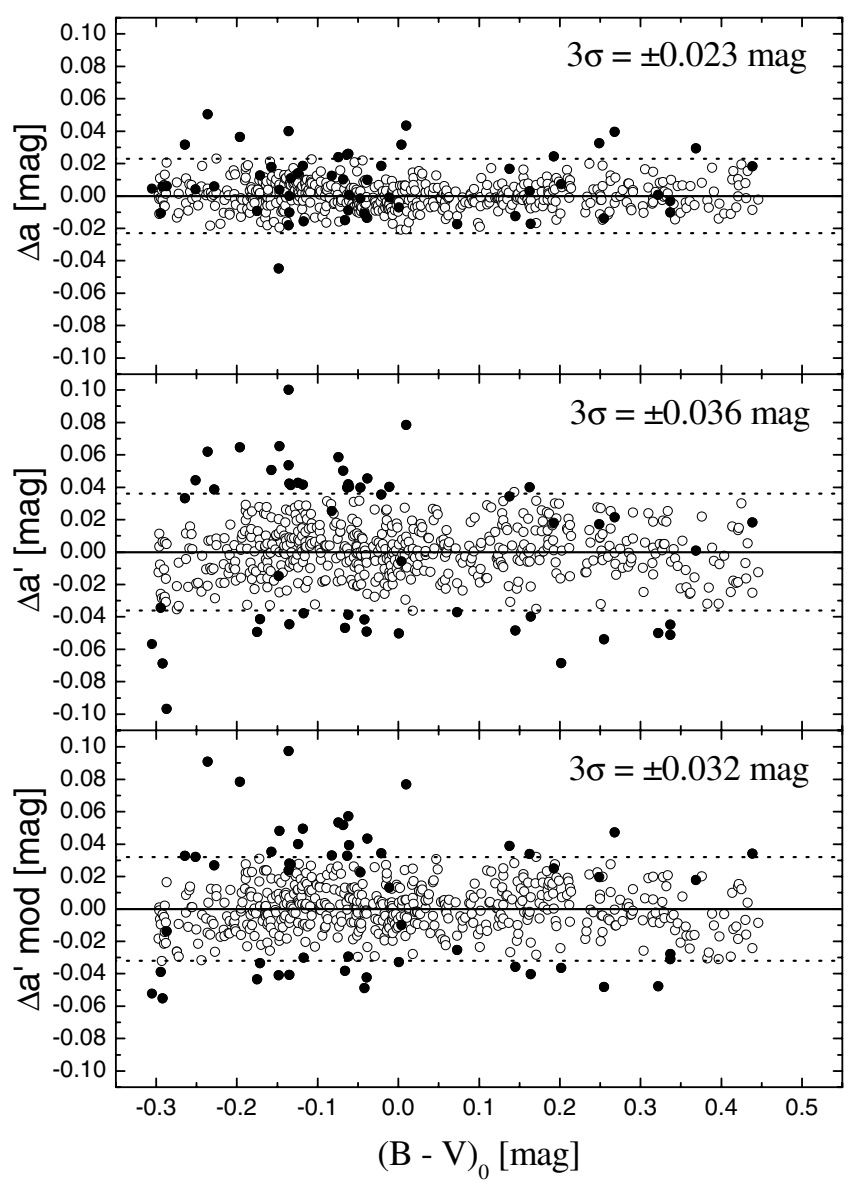

Fig. 2. Peculiarity indices of 631 stars in the spectral-class range from $\mathrm{O} 6$ to F5. The filled circles denote stars that are detected as peculiar within at least one index.

The three $\Delta a$ indices were calculated by subtracting the corresponding $a_{0}, a_{0}^{\prime}$ and $a_{0}^{\prime} \bmod$ from the $a, a^{\prime}$ and $a^{\prime} \bmod$ values for the same $(B-V)_{0}$. Figure 1 shows the peculiarity indices versus $(B-V)_{0}$. All stars above the threshold of $3 \sigma$ are expected to be chemically peculiar, whereas those below are emission-type and/or metal-weak objects. The region of $(B-V)_{0}$ in which CP2 stars are expected (hotter than spectral type F5) contains 631 stars (Fig. 2).

At $(B-V)_{0}$ of about $1.5 \mathrm{mag}$, corresponding to a spectral type M0, the indices $a, a^{\prime}$ and $a^{\prime} \bmod$ lose their correlation with $(B-V)_{0}$ although they are nearly linearly correlated at bluer colour indices. This may indicate a dependency of $a, a^{\prime}$ and $a^{\prime}$ mod on the luminosity class of the stars (see Fig. 3).

\section{Results}

The findings of our investigation are summarized in Tables 1 and 2. The first table lists all well-known CP stars taken from the catalogue by Renson \& Manfroid (2009). The second table includes apparently normal-type objects (according to spectral classification) hotter than spectral type F5 that have $\Delta a$ values both higher and lower than the $3 \sigma$ thresholds. The tables contain the HD number, spectral type, the $\Delta a$ values of each applied method, and the CP class (Table 1 only) of the stars. For comparison with existing peculiarity measurements, they also include published $\Delta a$ values (Paunzen et al. 2005) and $\Delta(V 1-G)$ values. The $\Delta(V 1-G)$ index is a measurement for peculiarity derived 


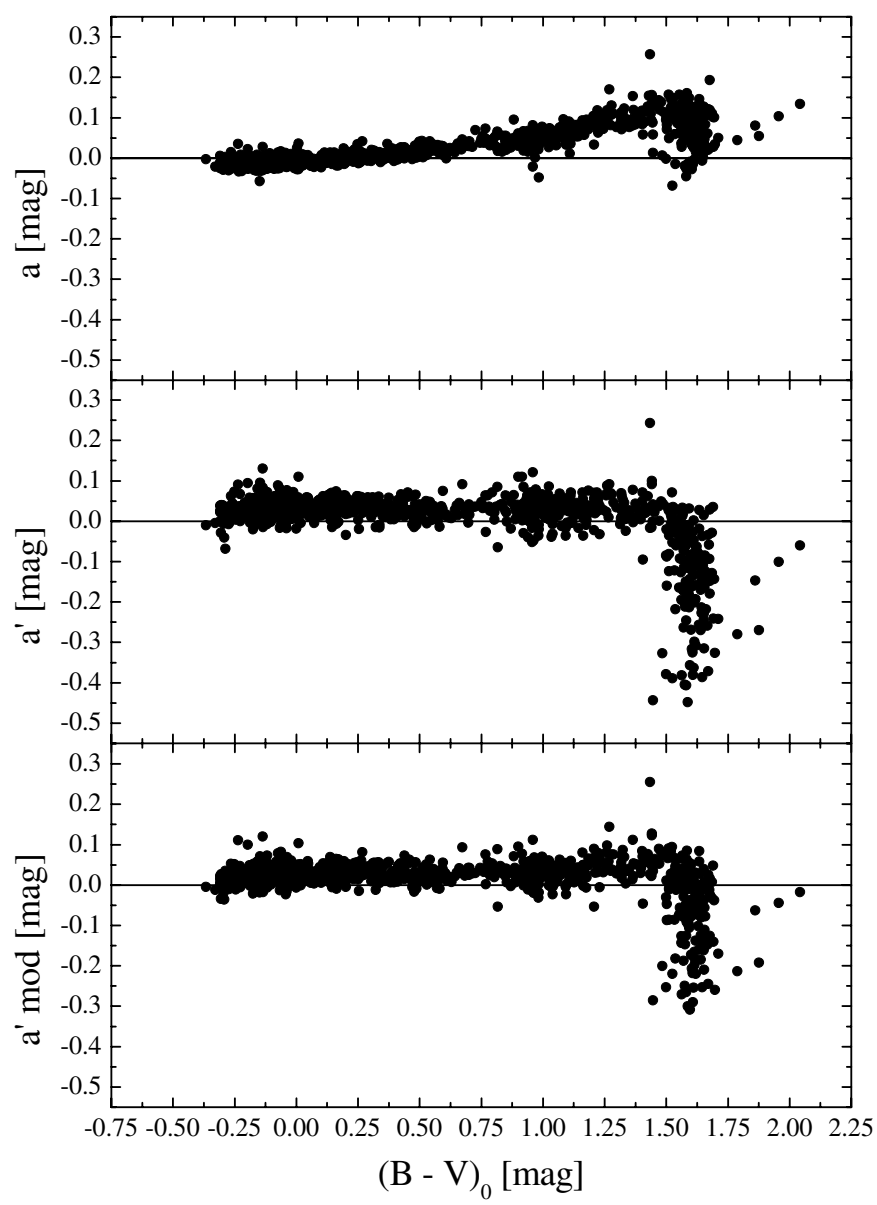

Fig. 3. Different $a$ indices of 1067 stars from the catalogue by Kharitonov et al. (1988) plotted versus $(B-V)_{0}$.

from the Geneva photometric system. Hauck (1974) was the first to propose an index as peculiarity parameter:

$\Delta(V 1-G)=(V 1-G)-0.289(B 2-G)+0.302$.

On average, normal stars have $\Delta(V 1-G)$ values of $-5 \mathrm{mmag}$, therefore we added +5 mmag to the calculated values. The photometric data of the Geneva system were collected by using the GCPD.

\subsection{Known peculiar objects}

First of all, we analysed the $55 \mathrm{CP}$ stars listed in Table 1 that are included in the catalogue by Renson \& Manfroid (2009). Altogether, 19 stars were detected beyond the $3 \sigma$ threshold by any of the systems. The modified system of Adelman yields 18 stars above $3 \sigma$ for this sample. This is the highest ratio of spectrophotometric to otherwise identified peculiar stars of the three applied detection methods.

In Fig. 4 we present the observed $\Delta(V 1-G)$ versus the synthetic $\Delta a^{\prime}$ mod values for our sample. The observed $\Delta a$ values were not used because there are too few available. There is a clear correlation of the observed and synthesized values. The five outliers (HD 11415, HD 115735, HD 189849, HD 201601, and HD 206088) are described below.

The CP1 (Am) stars have, with some rare exceptions, observed $\Delta a$ values well below +10 mmag and are normally

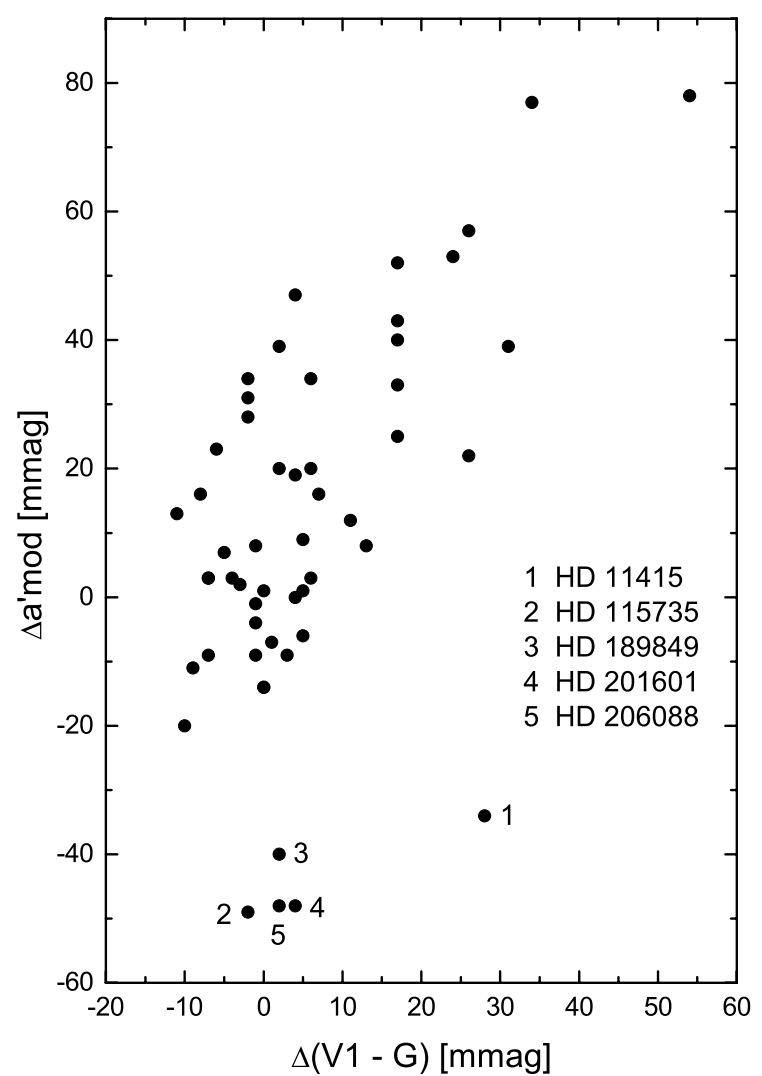

Fig. 4. Observed $\Delta(V 1-G)$ versus synthetic $\Delta a^{\prime} \bmod$ values $(\Delta a$ and $\Delta a^{\prime}$ behave very similar) for the $\mathrm{CP}$ star sample. The outliers are discussed in the text.

inconspicuous in the $\Delta(V 1-G)$ index. However, we detected four stars, three above and one below, the $3 \sigma$ threshold; these are

- HD 29479: a moderate overabundance of Fe-peak elements and $[\mathrm{Ba} / \mathrm{H}]=+1.77$ dex compared with that of the Sun was reported by Iliev et al. (2006).

- HD 76756: $\alpha$ Cancri is one of the prototype Am stars, with no other conspicuous features.

- HD 173648: this is a hot Am star with overabundances of most Fe-peak elements, and considerable overabundances of $\mathrm{Sr}, \mathrm{Y}, \mathrm{Zr}$, and $\mathrm{Ba}$ as well as some rare earths (Adelman et al. 1999).

- HD 189849: a weak magnetic field of about $250 \mathrm{G}$ was detected on a $20 \sigma$ level (Bychkov et al. 2009), so it might be misidentified.

It seems that some very peculiar CP1 stars are detectable via spectrophotometric observations and our peculiar indices.

The magnetic CP2 stars are mainly detectable via $\Delta a$ photometry, which is reflected in our results. Figure 5 shows the spectrophotometric data of standard star HD 102647 (A3 V) and HD 118022 (A2 Cr Eu Sr) for the whole spectral range (upper panel) and the region where the $\Delta a$ system is situated (lower panel). The UV excess (Sokolov 2006) and the $5200 \AA$ depression, both typical for CP stars, are clearly visible for HD 118022. All strong positive photometric detections are reproduced by our synthetic investigation. There are two objects, that have significant negative synthetic $\Delta a$ values, but have statistically insignificant observational values:

- HD 201601: $\gamma$ Equulei is a well-studied rapidly oscillating Ap star with a strong magnetic field and a very peculiar abundance pattern (Perraut et al. 2011). 
Ch. Stigler et al.: Spectrophotometric analysis of the $5200 \AA$ A region for peculiar and normal stars

Table 1. Synthetic $\left(\Delta a, \Delta a^{\prime}\right.$, and $\left.\Delta a^{\prime} m o d\right)$ and observed ( $\Delta a o b s$ and $\Delta(V 1-G)$ ) peculiarity indices in mmags for well-established CP (flag “*”) stars taken from Renson \& Manfroid (2009).

\begin{tabular}{|c|c|c|c|c|c|c|c|}
\hline HD & SpType & $\Delta a$ & $\Delta a^{\prime}$ & $\Delta a^{\prime} \bmod$ & $\Delta a o b s$ & $\Delta(V 1-G)$ & $\mathrm{CP}$ group \\
\hline 6116 & A3-A9 & +8 & +20 & +16 & - & -8 & 1 \\
\hline 20320 & $\mathrm{~A} 2-\mathrm{A} 9$ & -6 & -13 & -14 & -3 & 0 & 1 \\
\hline 27045 & $\mathrm{~A} 3-\mathrm{F} 3$ & -3 & +1 & -4 & - & -1 & 1 \\
\hline 27962 & $\mathrm{~A} 1-\mathrm{A} 4$ & +13 & +26 & +31 & - & -2 & 1 \\
\hline 28355 & A5-F1 & -1 & +2 & +2 & - & -3 & 1 \\
\hline 29140 & $\mathrm{~A} 3-\mathrm{A} 7$ & +12 & +25 & +23 & - & -6 & 1 \\
\hline 29479 & A3-A9 & +6 & +37 & +28 & - & -2 & 1 \\
\hline 40536 & $\mathrm{~A} 4-\mathrm{F} 1$ & -3 & +18 & +16 & +7 & +7 & 1 \\
\hline 41357 & $\mathrm{~A} 4-\mathrm{F} 2$ & -4 & +6 & -1 & - & -1 & 1 \\
\hline 76756 & A3-F1 & +17 & +34 & +39 & - & +2 & 1 \\
\hline 116657 & $\mathrm{~A} 1-\mathrm{A} 7$ & -6 & +19 & +25 & +10 & - & 1 \\
\hline 125337 & $\mathrm{~A} 2-\mathrm{A} 7$ & -11 & -8 & -9 & - & -7 & 1 \\
\hline 141795 & $\mathrm{~A} 2-\mathrm{A} 8$ & -1 & -6 & +3 & - & -7 & 1 \\
\hline 173648 & $\mathrm{~A} 4-\mathrm{F} 1$ & +3 & +40 & +34 & - & -2 & 1 \\
\hline 173654 & $\mathrm{~A} 2-\mathrm{A} 7$ & +3 & +17 & +13 & - & -11 & 1 \\
\hline 189849 & A5-A9 & -17 & -40 & -40 & - & +2 & 1 \\
\hline 197461 & $\mathrm{~A} 7-\mathrm{F} 0 \mathrm{dD}$ & -6 & -10 & -7 & - & +1 & 1 \\
\hline 198743 & A3-F3 & +8 & +5 & -9 & - & -1 & 1 \\
\hline 207098 & $\mathrm{~A} 5-\mathrm{F} 4 \mathrm{dD}$ & -4 & +7 & +1 & - & +5 & 1 \\
\hline 223461 & A3-F0 & +3 & +7 & +8 & - & -1 & 1 \\
\hline 10221 & A0 Si Sr Cr & +12 & +25 & +33 & - & +17 & 2 \\
\hline $11502 / 3$ & $\mathrm{~A} 1 \mathrm{Si} \mathrm{Cr} \mathrm{Sr}$ & +10 & +45 & +43 & +39 & +17 & 2 \\
\hline 15089 & $\mathrm{~A} 4 \mathrm{Sr}$ & -1 & -4 & 0 & - & +4 & 2 \\
\hline 19832 & B8 Si & +14 & +43 & +40 & +10 & +17 & 2 \\
\hline 26571 & B8 Si & +2 & -25 & -20 & +14 & -10 & 2 \\
\hline 32549 & B9 Si Cr & +10 & +50 & +52 & +25 & +17 & 2 \\
\hline 32650 & B9 Si & +8 & +12 & +12 & - & +11 & 2 \\
\hline 34452 & B9 Si & +36 & +65 & +78 & - & +54 & 2 \\
\hline 40312 & $\mathrm{~A} 0 \mathrm{Si}$ & +7 & +30 & +25 & - & +17 & 2 \\
\hline 68351 & $\mathrm{~A} 0 \mathrm{Si} \mathrm{Cr}$ & +24 & +58 & +53 & +24 & +24 & 2 \\
\hline 90569 & $\mathrm{~A} 0 \mathrm{Sr} \mathrm{Cr} \mathrm{Si}$ & +26 & +42 & +57 & +36 & +26 & 2 \\
\hline 112185 & $\mathrm{~A} 1 \mathrm{Cr} \mathrm{Eu} \mathrm{Mn}$ & +18 & +35 & +34 & - & +6 & 2 \\
\hline 112413 & $\mathrm{~A} 0 \mathrm{Eu} \mathrm{Si} \mathrm{Cr}$ & +13 & +19 & +22 & +40 & +26 & 2 \\
\hline 118022 & $\mathrm{~A} 2 \mathrm{Cr} \mathrm{Eu} \mathrm{Sr}$ & +43 & +78 & +77 & +51 & +34 & 2 \\
\hline 120198 & $\mathrm{~A} 0 \mathrm{Eu} \mathrm{Cr} \mathrm{Sr}$ & +1 & +40 & +39 & +38 & +31 & 2 \\
\hline 137909 & A9 $\mathrm{Sr} \mathrm{Eu} \mathrm{Cr}$ & +39 & +21 & +47 & +25 & +4 & 2 \\
\hline 148112 & $\mathrm{~A} 0 \mathrm{Cr} \mathrm{Eu}$ & -6 & +7 & +8 & - & +13 & 2 \\
\hline 170000 & $\mathrm{~A} 0 \mathrm{Si}$ & +7 & +6 & +6 & - & - & 2 \\
\hline 183056 & B9 Si & +13 & +23 & +20 & - & +2 & 2 \\
\hline 201601 & A9 $\mathrm{Sr} \mathrm{Eu}$ & -14 & -54 & -48 & +10 & +4 & 2 \\
\hline 206088 & A7-F3 Sr & +1 & -50 & -48 & +7 & +2 & 2 \\
\hline 358 & B9 Mn Hg & 0 & -7 & -6 & - & +5 & 3 \\
\hline 23950 & B9 Mn Hg Si & +3 & -19 & -14 & - & 0 & 3 \\
\hline 33904 & B9 Hg Mn & -1 & +3 & +3 & - & -4 & 3 \\
\hline 35497 & $\mathrm{~B} 8 \mathrm{Cr} \mathrm{Mn}$ & +3 & +4 & +3 & - & -7 & 3 \\
\hline 77350 & B9 $\mathrm{Sr} \mathrm{Cr} \mathrm{Hg}$ & -4 & +10 & +9 & +1 & +5 & 3 \\
\hline 78316 & B8 Mn Hg & 0 & +18 & +20 & +12 & +6 & 3 \\
\hline 106625 & B8 $\mathrm{Hg} \mathrm{Mn}$ & +3 & -18 & -11 & - & -9 & 3 \\
\hline 129174 & B9 $\mathrm{Mn} \mathrm{Hg}$ & -4 & +4 & +1 & - & 0 & 3 \\
\hline 143807 & A0 Mn Hg & -7 & +13 & +3 & - & +6 & 3 \\
\hline 145389 & B9 $\mathrm{Mn} \mathrm{Hg}$ & +15 & +8 & +19 & - & +4 & 3 \\
\hline 220933 & A0 $\mathrm{Hg} \mathrm{Mn}$ & -3 & -2 & +7 & - & -5 & 3 \\
\hline 11415 & B3 He wk. & +13 & -41 & -34 & - & +28 & 4 \\
\hline 23408 & B7 He wk. Mn & +13 & -9 & -9 & +5 & +3 & 4 \\
\hline 115735 & B9 He wk. & -11 & -42 & -49 & +2 & -2 & 4 \\
\hline
\end{tabular}

Notes. The synthetic photometric values for detected objects are given in boldface italics.

- HD 206088: a very peculiar object, in a transition between $\mathrm{CP} 1$ and $\mathrm{CP} 2$ shows strong variability in the infrared (Catalano et al. 1998).

The spectrophotometry of both objects might be severely influenced by the unusual elemental peculiarity and the variability.
Among the apparent non-magnetic CP3 (HgMn) stars, no detection in any system was found. This is perfectly in line with the results published by Paunzen et al. (2005).

Our sample includes only three well-known CP4 stars of which two (HD 11415 and HD 115735) show significant negative synthetic $\Delta a^{\prime}$ and $\Delta a^{\prime}$ mod values. Both objects are known to 
Table 2. Synthetic $\left(\Delta a, \Delta a^{\prime}\right.$, and $\left.\Delta a^{\prime} m o d\right)$ and observed ( $\Delta a$ obs and $\left.\Delta(V 1-G)\right)$ peculiarity indices in mmags for apparent non-CP stars detected via synthetic photometry.

\begin{tabular}{|c|c|c|c|c|c|c|}
\hline HD & SpType & $\Delta a$ & $\Delta a^{\prime}$ & $\Delta a^{\prime} \bmod$ & $\Delta a o b s$ & $\Delta(V 1-G)$ \\
\hline 5394 & B0.5 IVe & +17 & -37 & -21 & +5 & +1 \\
\hline 6811 & $\mathrm{~B} 7 \mathrm{Ve}$ & -10 & -45 & -41 & -5 & +2 \\
\hline 18552 & $\mathrm{~B} 8 \mathrm{Ve}$ & +40 & +100 & +97 & - & -2 \\
\hline 22192 & $\mathrm{~B} 5 \mathrm{Ve}$ & -9 & -49 & -44 & - & -4 \\
\hline 23016 & B8V (e) & -16 & -38 & -30 & - & -6 \\
\hline 32537 & F1 Vp MgII 4481 Å weak & -10 & -51 & -31 & - & -7 \\
\hline 34078 & O9.5 Ve, var. & +4 & -57 & -52 & - & -10 \\
\hline 35439 & B1 Vpe & -11 & -34 & -49 & +5 & +3 \\
\hline 67934 & A0 Vnp MgII 4481 Å weak & -1 & +40 & +13 & - & -4 \\
\hline 74873 & $\mathrm{kA} 0.5 \mathrm{hA} 5 \mathrm{~mA} 0.5 \mathrm{~V} \lambda \mathrm{Boo}$ & -18 & -37 & -25 & - & -15 \\
\hline 111604 & A5 Vp $\lambda$ Boo & -11 & -34 & -39 & - & -2 \\
\hline 112014 & A0 IIsp Mg,Si weak & -9 & -39 & -30 & - & +2 \\
\hline 193237 & B1 ep & +6 & -97 & -14 & +25 & +17 \\
\hline 209409 & B7 Ive & -18 & +53 & +24 & - & +4 \\
\hline 210839 & O6 If(n)p(e) & +6 & -69 & -55 & - & -19 \\
\hline 217891 & B6 IIIe & +4 & +65 & +48 & -1 & +4 \\
\hline 17769 & B7 V & +18 & +41 & +49 & - & -3 \\
\hline 19374 & B1 V $\beta$ Cep & +50 & +62 & +91 & - & -5 \\
\hline 22951 & A1 Vn & +32 & +33 & +33 & - & -7 \\
\hline 24554 & A1 V & +32 & -6 & -10 & - & - \\
\hline 28052 & F0 V & +32 & +17 & +19 & - & +6 \\
\hline 28149 & B5 V & +42 & +28 & - & - & \\
\hline 29248 & B2 III $\beta$ Cер & +4 & +44 & +32 & - & -9 \\
\hline 35671 & B5 V SB & +18 & +51 & +35 & - & -5 \\
\hline 35770 & B9.5 Vn & +26 & +40 & +33 & - & -4 \\
\hline 70011 & B9.5 V & -2 & +40 & +22 & - & +2 \\
\hline 76582 & A7 V & +24 & +18 & +25 & - & -6 \\
\hline 83808 & F8-G0III + A7m & +18 & +18 & +34 & - & -10 \\
\hline 107700 & G7III + A3IV & +29 & +1 & +18 & - & -4 \\
\hline 119765 & A0 V & -7 & -50 & -33 & +1 & - \\
\hline 139891 & B6V SB2 & +11 & +41 & +28 & - & - \\
\hline 166182 & B2 IV & +6 & +38 & +27 & - & -3 \\
\hline 178596 & F2 IV-V & -3 & -45 & -28 & - & -8 \\
\hline 188260 & B9.5 III & -15 & -47 & -38 & - & - \\
\hline 188350 & A0 III & -13 & -49 & -42 & - & -8 \\
\hline 212120 & B6 V, ell. var. & -45 & -15 & -41 & - & -2 \\
\hline 222603 & A7 V & +7 & -69 & -36 & -5 & -4 \\
\hline
\end{tabular}

Notes. The synthetic photometric values for detected objects are given in boldface italics. The upper panel lists well-known emission-type and metal-weak objects.

have strong emission lines according to Kohoutek \& Wehmeyer (1999) which explains our result.

\subsection{Apparent normal-type objects}

Table 2 lists the apparently non-CP stars detected via synthetic photometry. This sample can be divided into emission-type and metal-weak objects as well as inconspicuous stars.

The hot-emission-type stars are defined as dwarfs that have shown hydrogen emission in their spectra at least once. Due to a developed equatorial disk that is produced by stellar winds, emission arises quite regularly. In addition, photometric variability on different time scales is a common phenomenon caused by the formation of shock waves within those disks. The phases of emission are replaced by shell and normal phases of the same object, leading to a transition from negative to positive $\Delta a$ values (Pavlovski \& Maitzen 1989). Among the metalweak objects, the most prominent subgroup are the $\lambda$ Bootis stars. This small group of objects comprises late-B to earlyF-type stars, with moderate to extreme (up to a factor 100) surface under-abundances of most Fe-peak elements and solar abundances of lighter elements ( $\mathrm{C}, \mathrm{N}, \mathrm{O}$, and $\mathrm{S})$. The main mechanisms responsible for this phenomenon are atmospheric diffusion, meridional mixing, and accretion of material from their surroundings (Paunzen et al. 2002). The $\Delta a$ observations of the two groups are summarized in Paunzen et al. (2005).

In the sample of inconspicuous objects, we detected two $\beta$ Cephei pulsators (HD 19374 and HD 29248) with significantly high positive $\Delta a$ values. These objects are early-type-B stars with light and radial velocity variations on time scales of several hours (Stankov \& Handler 2005). Since these objects are quite rare, fewer than 200 Galactic objects are known, it would be interesting to find out whether they can be detected via $\Delta a$ photometry. Unfortunately, no observations in this respect have been performed so far.

The remaining objects have two characteristics in common:

1. Except for one object (HD 22951), all of them are very fast rotators $\left(v \sin i \geq 150 \mathrm{~km} \mathrm{~s}^{-1}\right)$ which is atypical for CP stars.

2. Almost all objects are in binary systems, which might distort the spectrophotometry. 

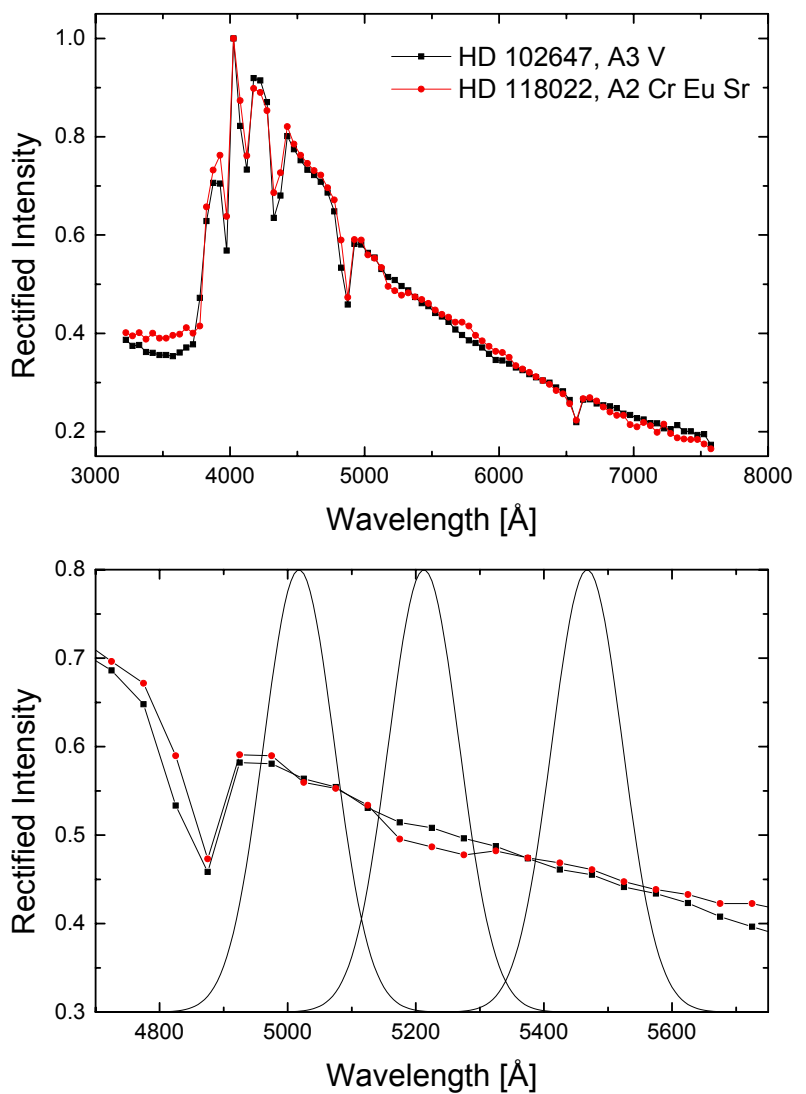

Fig. 5. Spectrophotometric data of HD 102647 (A3 V) and HD 118022 (A2 $\mathrm{Cr} \mathrm{Eu} \mathrm{Sr}$ ) for the whole spectral range (upper panel) and the region where the $\Delta a$ system is situated (lower panel). The UV excess and the $5200 \AA$ depression, both typical for CP stars, are clearly visible for HD 118022.

For the following objects we found additional interesting characteristics in the literature:

- HD 24554: Schröder \& Schmitt (2007) found strong X-ray emission indicating an undetected binary nature of the object.

- HD 28052: this is the second-brightest X-ray source in the Hyades and a possibly quadruple system with at least one component of $\delta$ Scuti type (Simon \& Ayres 2000).

- HD 83808: this spectroscopic binary system is listed as CP1 candidate in Renson \& Manfroid (2009).

- HD 107700: Griffin \& Griffin (2011) analysed this closebinary system and found a slight metal-weakness for both components.

- HD 119765: Renson \& Manfroid (2009) listed it as questionable $\mathrm{CP}$ candidate without any designation to a specific subgroup.

For these samples, $\Delta a^{\prime}$ is superior to the other two systems. For almost all of the newly discovered peculiar objects among the normal-type objects, photometric $\Delta a$ and/or spectroscopic observations are needed to clarify their nature.

We also identified nine cool-type objects $(0.5<(B-$ $V)_{0}<1.5 \mathrm{mag}$ ) that significantly deviate in the positive direction. Those stars are:

- HD 19373: Canto Martins et al. (2011) analysed the chromospheric activity of the G0 V object.

- HD 26965: this is a young triple binary system including flare-type objects (Pettersen 1991).
- HD 35369: Prugniel et al. (2011) found an underabundance of $[\mathrm{Fe} / \mathrm{H}]=-0.22$ dex compared with the Sun.

- HD 49878: there are no detailed investigations for this K-type giant available in the literature.

- HD 68375: Takeda et al. (2008) investigated it in more detail, and found no peculiarities.

- HD 82635: this is a highly chromospherically active RS CVn type giant (Strassmeier et al. 1994).

- HD 158899: according to Antipova et al. (2004), this is a moderate Barium star classified as K3.5 III Ba0.1. This group of chemically peculiar stars consists of $\mathrm{G}$ to $\mathrm{K}$ giants, whose spectra indicate an overabundance of s-process elements.

- HD 192577: it is a $\zeta$ Aurigae type eclipsing binary of spectral type K4 I (Eaton 2008).

- HD 194093: Gray (2010) presented photometric and spectroscopic time series of this variable star. The variations are found on all time scales up to several hundred days.

We found that the $a$ indices are linearly correlated with the effective temperature up to a spectral type of M0. For cooler-type objects, there is a strong indication of an additional luminosity effect that is superimposed on the temperature dependency. Up to now, no photometric $\Delta a$ data of cool-type stars have been published. Our findings suggest that such observations will be very interesting for the study of low-mass peculiar objects.

\section{Conclusions and outlook}

We used the spectrophotometric data of the stellar catalogue by Kharitonov et al. (1988) to synthesize three different " $a$ systems". These data cover the complete spectral range from low- to high-mass objects. For the first time, we presented $a$ indices for stars cooler than F-type. Excluding low-quality data and earlytype supergiants, 1067 stars were used to synthesize different peculiarity indices. Our main results are:

- most of the known classical magnetic chemically peculiar stars were detected;

- we presented a list of about 50 normal-type objects across the complete spectral range that were detected;

- the most efficient $a$ system is very similar to that previously employed by Maitzen (1980);

- the normality line of the $a$ system correlates with the effective temperature up to a spectral type of M0.

Our analysis showed that spectrophotometric data can be used for calculating synthetic $a$ indices and for detecting peculiar objects across the complete spectral range up to M0. As next steps, we will observe cool-type objects to establish the corresponding correlations. In addition, we will use classification resolution spectra (for example from LAMOST) to search for chemically peculiar objects in a semi-automatic way.

Acknowledgements. This project is financed by the SoMoPro II programme (3SGA5916). The research leading to these results has acquired a financial grant from the People Programme (Marie Curie action) of the Seventh Framework Programme of EU according to the REA Grant Agreement No. 291782. The research is further co-financed by the South-Moravian Region. It was also supported by the grants GA ČR P209/12/0217, 14-26115P, 7AMB12AT003, and the financial contributions of the Austrian Agency for International Cooperation in Education and Research (BG-03/2013 and CZ-10/2012). This research has made use of the WEBDA database, operated at the Department of Theoretical Physics and Astrophysics of the Masaryk University. This work reflects only the author's views and the European Union is not liable for any use that may be made of the information contained therein. 


\section{References}

Adelman, S. J. 1979, AJ, 84, 857

Adelman, S. J., Pyper, D. M., Shore, S. N., White, R. E., \& Warren, W. H., Jr. 1989, A\&AS, 81, 221

Adelman, S. J., Caliskan, H., Cay, T., Kocer, D., \& Tektanali, H. G. 1999, MNRAS, 305, 591

Antipova, L. I., Boyarchuk, A. A., Pakhomov, Yu. V., \& Panchuk, V. E. 2004, Astron. Rep., 48, 597

Babcock, H. W. 1947, ApJ, 105, 105

Burnashev, V. I. 1985, Abastumanskaya Astrofiz. Obs., Byull., 59, 83

Bychkov, V. D., Bychkova, L. V., \& Madej, J. 2009, MNRAS, 394, 1338

Canto Martins, B. L., das Chagas, M. L., Alves, S., et al. 2011, A\&A, 530, A73

Catalano, F. A., Leone, F., \& Kroll, R. 1998, A\&AS, 129, 463

Cramer, N. 1999, New Astron., 43, 343

Eaton, J. A. 2008, AJ, 136, 1964

Glagolevskij, Yu. V. 2013, Astrophysics, 56, 173

Glushneva, I. N., Kharitonov, A. V., Kniazeva, L. N., \& Shenavrin, V. I. 1992, A\&AS, 92, 1

Gray, D. F. 2010, AJ, 140, 1329

Griffin, R. E. M., \& Griffin, R. F. 2011, Astron. Nachr., 332, 105

Gutierrez-Moreno, A. 1975, PASP, 87, 805

Hauck, B. 1974, A\&A, 32, 447

Havnes, O., \& Conti, P. S. 1971, A\&A, 14, 1

Iliev, I. Kh., Budaj, J., Fenovcík, M., Stateva, I., \& Richards, M. T. 2006, MNRAS, 370, 819

Jordi, C., Carrasco, J. M., Fabricius, C., Figueras, F., \& Voss, H. 2010, Highlights of Spanish Astrophysics V (Berlin: Springer-Verlag), Astrophys. Space Sci. Proc., 147
Khan, S. A., \& Shulyak, D. V. 2007, A\&A, 469, 1083

Kharitonov, A. V., Tereshchenko, V. M., \& Knyazeva, L. N. 1988, The spectrophotometric catalogue of stars, Nauka, Alma-Ata (USSR)

Kodaira, K. 1969, ApJ, 157, L59

Kohoutek, L., \& Wehmeyer, R. 1999, A\&AS, 134, 255

Maitzen, H. M. 1976, A\&A, 51, 223

Maitzen, H. M. 1980, A\&A, 89, 230

Maitzen, H. M., \& Moffat, A. F. J. 1972, A\&A, 16, 385

Maitzen, H. M., \& Muthsam, H. 1980, A\&A, 83, 334

Mermilliod, J.-C., Mermilliod, M., \& Hauck, B. 1997, A\&AS, 124, 349

Napiwotzki, R., Schoenberner, D., \& Wenske, V. 1993, A\&A, 268, 653

Netopil, M., Paunzen, E., Maitzen, H. M., North, P., \& Hubrig, S. 2008, A\&A, 491, 545

Paunzen, E., Iliev, I. Kh., Kamp, I., \& Barzova, I. 2002, MNRAS, 336, 1030

Paunzen, E., Stütz, Ch., \& Maitzen, H. M. 2005, A\&A, 441, 631

Pavlovski, K., \& Maitzen, H. M. 1989, A\&AS, 77, 351

Perraut, K., Brandão, I., Mourard, D., et al. 2011, A\&A, 526, A89

Pettersen, B. R. 1991, Mem. Soc. Astron. It., 62, 217

Preston, G. W. 1974, ARA\&A, 12, 257

Prugniel, P., Vauglin, I., \& Koleva, M. 2011, A\&A, 531, A165

Renson, P., \& Manfroid, J. 2009, A\&A, 498, 961

Schröder, C., \& Schmitt, J. H. M. M. 2007, A\&A, 475, 677

Simon, T., \& Ayres, T. R. 2000, ApJ, 539, 325

Sokolov, N. A. 2006, MNRAS, 373, 666

Stankov, A., \& Handler, G. 2005, ApJS, 158, 193

Stift, M. J., \& Alecian, G. 2012, MNRAS, 425, 2715

Strassmeier, K. G., Handler, G., Paunzen, E., \& Rauth, M. 1994, A\&A, 281, 855

Takeda, Y., Sato, B., \& Murata, D. 2008, PASJ, 60, 781 